\title{
Teaching The Practice Of Compassion To Nursing Students Within An Online Learning Environment: A Qualitative Study Protocol
}

\author{
Anne Hofmeyer, University of South Australia, Australia \\ Luisa Toffoli, University of South Australia, Australia \\ Rachael Vernon, University of South Australia, Australia \\ Ruth Taylor, Anglia Ruskin University, United Kingdom \\ Dorrie Fontaine, University of Virginia, USA \\ Hester C. Klopper, Stellenbosch University, South Africa \\ Siedine Knobloch Coetzee, North-West University, South Africa
}

\begin{abstract}
Background: There is an increasing global demand for higher education to incorporate flexible delivery. Nursing education has been at the forefront of developing flexible online education and offering programs 'anywhere and anytime'. In response to calls to teach compassion in nursing education, there is an abundance of literature concerning classroom teaching, but few online studies.
\end{abstract}

Design: This paper presents a qualitative study protocol to explore the effectiveness of a compassion module taught to undergraduate nursing students within a digital learning environment. The protocol consists of an online knowledge intervention (compassion module) and pre- and post-intervention qualitative questions to explore and describe undergraduate nursing students' understanding and learning about the practice of compassion toward patients, colleagues and self.

Methods: Students will study the online compassion module over a two week period in a theory course in the final year of the Bachelor of Nursing. Students will participate in the study by responding to open-ended questions, administered through SurveyMonkey ${ }^{\circledR}$ before and after studying the online compassion module. Free text responses will be analysed thematically. The researchers will obtain institutional ethical approval.

Discussion: This study protocol may generate new knowledge about how nursing students learn compassionate practice within an online learning environment. These findings could be used by nurse leaders and educators to develop empirically supported curricula and workplace cultures to foster the practice of compassion and resilience in the next generation of nurses.

Keywords: Qualitative; Online Education; Compassion; Health Care; Nursing

\section{INTRODUCTION}

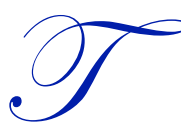

questo compassion toward patients, colleagues and self.

Copyright by author(s); $\underline{\mathrm{CC}-\mathrm{BY}}$ 
The importance of compassionate care to patients and families is well established (Bramley \& Matiti, 2014; Sinclair et al., 2016a; Sinclair et al., 2016b). Notably, 'cultivating a resilient healthcare workforce committed to high quality, compassionate, relationship-based care' (Bauer-Wu \& Fontaine, 2015, p.16) is championed globally, and is the cornerstone of many healthcare policies. Caring and being compassionate is the essence and moral foundation of healthcare (Gaut \& Leninger, 1991) nursing practice, and education (Nightingale 1859; Bevis \& Watson, 1989; Benner \& Wrubel, 1989; Leininger \& Watson, 1990). Moreover, compassion is as important as clinical competence in the delivery of quality healthcare (Davidson et al., 2013; van der Cingel, 2014). Organisational, team and individual factors operate to enable or hinder 'caring cultures in which compassionate care can flourish' (Christiansen et al., 2015, p. 837). Hence, raising undergraduate nursing students' awareness of these factors is, therefore, timely.

\section{Defining Compassion}

The concept of compassion has been used interchangeably with qualities such as empathy and sympathy (Richardson et al., 2015; Curtis et al., 2012; von Dietze \& Orb, 2000; Goetz et al., 2010). Empathy means understanding the feelings of another (Kenny, 2016). But compassion is different (Nassbaum, 2003; Schantz, 2007; Sinclair et al., 2016a). The intention to act to relieve the suffering of others distinguishes compassion from other virtuous qualities (Sinclair et al., 2016a; Maben et al., 2010; Papadopoulos \& Ali, 2016; Schantz, 2007). Notably, patients can clearly describe their experiences of compassionate nursing care or when they felt compassion was absent in their care (Bramley \& Matiti, 2014). Sinclair and colleagues (2016a, p. 193) have developed the first empirically based model of compassion, and define 'compassion as a virtuous response that seeks to address the suffering and needs of a person through relational understanding and action'. Implicit in this definition is the action to respond to the needs and suffering of a person, not just a general intention to care for others.

\section{Compassion is an Essential Ingredient in Quality Care}

The contribution of compassionate care to improve quality care, patient outcomes and nurse well-being is well documented (Bauer-Wu \& Fontaine, 2015; Aiken et al., 2014; Lown, 2014; Seppala et al., 2014; NHS Confederation, 2008). Patients and their families say the manner in which they are listened to and cared for with compassion is highly valued, and matters just as much as the quality of health care itself (Bray et al., 2014; Lown et al., 2011; Schout et al., 2009). Compassionate care addresses the patient's core need for 'connection and relationships and is based on attentive listening and a desire to understand the patient's context and perspective' (Lown et al., 2011). Its presence enhances effectiveness and person-centred care (Lown, 2014). Compassion can be felt through attentive listening, respect, and sensitivity to what is said and not said. It can 'ameliorate concerns, distress or suffering' (Lown, 2014, p. 6). But importantly, compassion is not only essential for excellent care of patients, but also for reducing burnout and improving health outcomes, well-being, and resilience in clinicians themselves (Bauer-Wu \& Fontaine, 2015; Seppala et al., 2014; Lamothe et al., 2014).

\section{Factors that Hinder or Support the Practice of Compassion in Organisational Cultures}

There is a global concern that, despite significant resources for health and a focus on improvement in evidence-based practice, health systems are still failing to meet the core holistic and compassionate care needs and expectations of patients (Bray et al., 2014; Lown, 2014; Lown et al., 2011). Reports indicate that communication and compassion are not consistent features in healthcare cultures that are under increasing strain (Aiken et al., 2014). International research has linked poor outcomes for patients with austerity measures and the perceived lack of compassion in healthcare (Bray et al., 2014). Recent concerns about the lack of compassion and poor quality patient care have focused on individual preparedness and the virtuous intent of nurses. However, organisational barriers such as unmanageable workloads, staff shortages, low team morale, indifferent management, inadequate training, and limited resources continue to hinder compassionate care and foster staff stress (Aiken et al., 2014; Lown, 2014; Seppala et al., 2014; Clark et al., 2013). Further, 'incivility in the healthcare workplace is pervasive: stressed professionals treat their colleagues with disrespect, thereby perpetuating the stressful work environment' (Bauer-Wu \& Fontaine, 2015, p 17). Stress compromises clinicians' clear thinking and clinical decision-making, thus increasing the risk of errors and threatening patient safety (Bauer-Wu \& Fontaine, 2015, p 17). We are reminded by Clark (2013, p. 17) about the cost of incivility in work environments and that 'disruptive and intimidating behaviours can have negative and lasting effects for individuals, teams and organisations' and results in staff turnover, toxic relationships and poor productivity. 
Addressing the organisational, team and individual factors that hinder or enable the delivery of compassion is complex and is a 'pivotal role for leaders' (Christiansen et al., 2015, p. 837). Arguably, a systems approach is needed to identify facilitators and barriers in organisational environments, rather than blaming individual providers for a lack of compassion in healthcare systems (Crawford et al., 2014; Gilbert, 2005). The influence of organisational structures and processes as facilitators or barriers to the presence and practice of compassion in healthcare environments warrants greater attention (McCaffrey \& McConnell, 2015). Improving compassionate healthcare provision is dependent on strengthening systems and people (Curtis, 2015). In sum, supportive environments facilitate the provision of compassionate care, although individuals remain accountable for their practice (Horsburgh \& Ross, 2013, p. 1124) and well-being.

\section{Compassion Fatigue}

The term compassion fatigue was first used by Joinson to describe nurses who had lost their 'ability to nurture' (1992 p. 119). However, fatigue is more than feeling tired. Demanding workplaces, barriers and incivility can result in compassion fatigue for individuals (Coetzee \& Klopper, 2010; Yoder 2010). Compassion fatigue can be understood as nurses 'losing their nurturing ability toward patients' (Coetzee \& Klopper, 2010), toward colleagues, and toward themselves. Compassion fatigue (emotional exhaustion, burnout, lack of compassion and connection) can negatively affect professional and personal relationships and work performance (Hunsaker et al., 2015; Berger et al., 2015; Beaumont et al., 2016). Further, clinicians experiencing burnout are more likely to depersonalize patients such as referring to them as their disease (Bauer-Wu \& Fontaine, 2015). More than 40 percent of nurses working in hospital environments report diminished resilience, physical, emotional or mental exhaustion (Sieg, 2015). Therefore, their ability to reflect on practice to provide compassionate care for patients and work as an effective, resilient team member is under threat (Aiken et al., 2014; Dewar et al., 2014).

\section{Self-Compassion and Resilience}

Research into compassion has focused on how individual nurses can provide compassionate care for patients and their families. But self-compassion must exist before individuals can practice compassion toward others. However, there is scant research on the topic of self-compassion (Mills et al., 2015; Izhaki et al., 2015). Self-compassion is about having feelings of kindness for oneself, as one would have for others (Neff 2003a). Self-care and kindness are essential to our well-being and the foundation for building resilience and practising compassion toward others. Resilience refers to a person's ability to thrive, continue to be positive, and function despite difficult circumstances and adversity (Campbell-Sills \& Stein, 2007). Moreover, Drury et al., (2014) have shown that resilient nurses are more likely to stay in nursing and their care is higher-quality. To care for others, nurses must care for themselves and nurture their resilience (McAllister \& McKinnon, 2009).

\section{Can Compassion Be Taught?}

There has been debate about whether compassion is an innate value or whether it can be taught (Richardson et al., 2015). The relationship between nursing students' personal attributes as a precursor to practicing compassion has also been investigated (Pitt et al., 2014). Thus, how this core professional value can be enacted and evaluated in practice and made explicit in nursing curricula (Adamson \& Dewar, 2011) deserves attention (Bray et al., 2014; Adam \& Taylor, 2013). Significant studies have investigated the role of higher education in preparing students to practice compassion in clinical settings (see, for example: Bauer-Wu \& Fontaine, 2015; Adam \& Taylor, 2013; Adamson \& Dewar, 2014; Adamson \& Dewar, 2011; Dewar et al., 2014; Curtis, 2013; Curtis, 2014; Cummings \& Bennett, 2012; Bray et al., 2014). Recent work has focused on methods to teach, simulate, and assess for compassion (Kenny, 2016), along with the validation of an instrument to measure nurses' compassion competence (Sinclair et al., 2016a Lee \& Seomun, 2016). Previous instruments only focused on communication, whereas compassion is about acting to relieve suffering (Sinclair et al., 2016a; Lee \& Seomun, 2016). However, Klopper (2015) cautions us to remember that not everything that counts in nursing can be measured.

Few, if any, studies have explored whether or not a digital learning environment can teach this core professional value. Digital learning is an umbrella term to define teaching practices where technology is used to support the learning 
process. It includes online, blended and mobile learning practices. Without such evidence, it is difficult for nurse educators to bridge the gap by determining curricula and the effectiveness of compassion education.

\section{Rationale}

Evidence has shown that compassion fatigue limits nurses' ability to care for patients (Izhaki et al., 2015; Coetzee \& Klopper, 2010). In view of this, it is important for nursing students to explore understandings of compassion and resilience to be effective in their professional practice. The challenge for nurse educators is to create innovative online curricula to teach compassion and resilience that nurses need to practice effectively compassion in demanding healthcare environments. This study will address this goal through the explicit teaching of compassion to undergraduate nursing students within an online learning environment. Our study is guided by the view that: 'Compassion is how care is given through relationships based on respect and dignity - it can also be described as intelligent kindness and is central to how people perceive their care' (Cummings \& Bennett, 2012).

\section{THE STUDY}

\section{Design}

The researchers will use a qualitative descriptive pre-post intervention design. The qualitative design is particularly suited to our aim of exploring the understanding of compassion and practices toward self and others by undergraduate nursing students (Sandelowski, 2010). This qualitative study will explore the effectiveness of an online compassion module taught to undergraduate nursing students within a digital learning environment. The study protocol consists of an online knowledge intervention (compassion module) and pre- and post-intervention qualitative questions to explore and describe undergraduate nursing students' understanding and learning about the practice of compassion toward patients, colleagues and self, (provided in Appendix 1). Students will study the knowledge intervention over a two week period in 'Nursing Project' which is a theory course taught in the final year of the Bachelor of Nursing Program. Students will take part in the study by responding to open-ended questions, administered through SurveyMonkey ${ }^{\circledR}$ before and after studying the knowledge intervention.

\section{Knowledge Intervention: Compassion Module}

The purpose of the compassion module is to educate students about the importance of compassion in healthcare; being compassionate toward patients, colleagues and self; cultivating resilience; and the organisational, team and individual factors that hinder or enable compassionate care. The compassion module poses eight questions and evidence-based responses. Reflective questions to raise students' awareness and key readings conclude each section. Also students will watch an 18 minute Compassionate Care video (Fontaine, 2012). In the video, nurses, physicians, administrators, professors and students talk about practicing compassion toward themselves, colleagues and patients, and how they strengthen their resilience through mindfulness.

\section{Pre- and Post-Intervention Qualitative Questions}

Qualitative surveys consist of a series of open-ended questions about a topic that can be completed online (Braun \& Clarke, 2013). Participants type responses in text boxes like open-ended survey questions. Online qualitative surveys can be an ideal method to access geographically dispersed participants (Polit \& Beck, 2011; Braun \& Clarke, 2013). In our study, 33 percent of students invited to participate were located across Australia and were enrolled by 'external' mode. So this method for collecting qualitative data was ideal for our study. The qualitative data will be collected before and after the students study the compassion module. The qualitative questions will be distributed via the electronic platform SurveyMonkey®. Text boxes will be used to collect open-ended, written responses.

\section{Cognitive Interviewing}

Non-response or non-completion of questionnaires is a major problem in survey research leading to the collection of incomplete data (Drennan, 2003). Cognitive interviewing is a pre-testing technique to identify problematic questions 
before administering the survey (Drennan, 2003). The aims of cognitive interviewing are to: understand how the questions are perceived and interpreted from the respondent's perspective, rather than that of the researcher; identify problems with wording and organization that could potentially lead to misunderstandings and missing data; and to increase response rates (Drennan, 2003). We conducted a cognitive interviewing session with eight students to provide feedback about the clarity of instructions, layout, wording and sequencing of the qualitative questions (Braun \& Clarke, 2013; Drennan, 2003; Hofmeyer et al., 2015). We made several changes to the pre- and post-intervention surveys based on the feedback, for example:

- Can you give an example of you (or someone else) being compassionate towards a client, resident or patient? (Pre-intervention: Q3; Post-intervention: Q3). Feedback confirmed that adding the words 'or someone else' increased the likelihood of eliciting a response. Some students said they would skip that question because they couldn't think of an example from their practice.

- Can you give an example of you (or someone else) practising compassion toward a colleague in the workplace? (Post-intervention: Q5). Similar feedback as above.

- Post-intervention: Q12 was originally worded: What is a key message that you learned in the module. The new wording became: What is one important idea that you learnt in the module.

- A few students thought Q13 (Thinking about what you have learnt, how could this knowledge influence your practice as a nurse?) was more comprehensive, so suggested deleting Q12.

- Pre-intervention: Q5; Post-intervention: Q15 was originally worded: What ten (10) key words summarise this topic for you? Feedback to delete the word 'key' and to request six summary words.

Researchers implementing this study protocol may wish to conduct a cognitive interviewing session with a representative sample of participants to ensure the materials are coherent and relevant in the local context.

\section{Ethical Considerations}

We will obtain ethical approval to conduct the study from the University institutional ethics committee (IEC). Submission of participant responses through SurveyMonkey ${ }^{\circledR}$ will imply consent to participate in the study. All deidentified data will be stored securely, according to ethical guidelines.

\section{Recruitment}

We will send an introductory email to all undergraduate nursing students enrolled in 'Nursing Project' to invite them to participate in the study. Attached will be the IEC approved participant information letter to introduce the investigators, explain the study, and address confidentiality, anonymity, informed consent, secure storage of data, and IEC contact details. The email will contain a link to the set of open-ended questions administered via SurveyMonkey®. Students will receive a reminder email seven and fourteen days after the introductory email. This schedule will guide the pre-post data collection.

\section{Data Collection}

We will collect data at two distinct time points:

- Pre-intervention: five open-ended questions to explore and describe participants' understandings about compassion and practicing compassion in nursing and seven demographic questions;

- Post-intervention: twelve open-ended questions to explore and describe participants' learning and understanding about compassion, practicing compassion, self-compassion, resilient strategies, ideas to improve the module and seven demographic questions.

\section{Data Analysis}

Participant demographics including age, gender, enrolment profile and past educational experience will be analysed using descriptive statistics. Qualitative analysis of the free text responses will be conducted manually and 
independently by the investigators. This thematic, inductive approach will involve multiple readings of the responses and labelling relevant sections or words to understand participants' meanings and analyse similar and different patterns (Borbasi \& Jackson, 2016). Identifying the core meanings in the text relevant to the research objectives and survey questions will guide analysis. We will engage to compare emergent findings and differences will be resolved by consensus. Significant phrases will be identified and categorized into preliminary themes (Braun \& Clark, 2013; Ruona, 2005). We will perform code frequency counts to identify the most frequently reported words and themes. The researchers will order preliminary themes until themes and sub-themes are confirmed, and findings are formulated (Borbasi \& Jackson, 2016). Participant quotations to illustrate themes will be selected. We will explore how the themes are connected to each other and to the literature to confirm resonance.

\section{Rigour and Trustworthiness}

We will assure trustworthiness rather than generalisability in this qualitative study. Our systematic approach will support the rigor and trustworthiness (Lincoln \& Guba, 1985), dependability and credibility of our qualitative study (Polit \& Beck, 2011). To ensure trustworthiness of the data, each researcher has the qualifications and practical experience and skills to conduct the study (Patton, 1990). The reflexive process will be documented to provide a clear audit trail of the rationale for decision-making throughout the study. The researchers will establish authenticity and confirmability through the use of participant quotations and a careful synthesis of the perspectives to support the findings and ensure conclusions are well grounded in the data. The rigorous data analysis process, the researchers' expertise, and audit trail of decisions will ensure trustworthiness of the data (Ruona, 2005; Guba \& Lincoln, 1994). The qualitative questions and compassion module were tested using the cognitive interviewing process and reviewed by an experienced investigator. Publication and presentation of findings for target audiences such as the study participants, researchers, nurse educators and other relevant stakeholders will provide opportunities to confirm resonance and credibility (Lincoln \& Guba, 1985). There is scope for researchers to assess transferability and applicability of this study protocol for other contexts.

\section{CONCLUSION}

Caring for others has a cost, so nurses' self-care and resilience is paramount. It is essential to educate undergraduate nursing students in their responsibility to enhance their resilience to practice compassion and foster better outcomes for patients. McDonald et al. (2016, p. 129) remind us:

Nursing management must ensure new graduates and early career nurses and midwives are aware of the potential effects of workplace adversity on their health and well-being. Nursing graduates may be forewarned of the potential dangers of professional burnout and may have practised self-reflection techniques; they should also understand how developing personal resilience could assist them to meet the demands of their careers.

All nurses have a part to play to address the professional practice crisis. Organisational cultural change must go hand in hand with recruiting nurses with high levels of compassion and resilience (McPherson et al., 2016). A stronger focus is vital to foster civility in nursing (Clark, 2013), get the basics right in education (Darbyshire \& McKenna, 2013) and cultivate environments that improve professional socialization and foster compassionate care for self and others (Christiansen et al., 2015; Curtis et al., 2012; Beaumont et al., 2016).

This study protocol has been designed to address this priority through the explicit online teaching of compassion to foster effective professional practice, self-compassion, resilience, and self-responsibility in the next generation of nurses. We recommend this study protocol could be implemented in any undergraduate nursing program and the qualitative pre - post questions could be used to conduct individual or focus group interviews with nursing students. The technology to support the delivery of flexible online education is well developed and accessible. Findings would contribute to the emergent literature about how compassion may be taught in nursing.

As Maya Angelou (2014) said: "I've learned that people will forget what you said, will forget what you did, but they will never forget how you made them feel". 
Better prepared nurses with self-awareness will practice compassion with patients, families and colleagues. Nurses and managers' insight, skills and resilience will influence the organisational factors to foster compassion in healthcare workplaces. This study protocol may be vital step to foster compassionate practices and cultures; thereby better serving the patients and families in our care - surely our raison d'être as a profession.

\section{ACKNOWLEDGEMENTS}

Funding: Funding was provided by the Nurses' Memorial Foundation of South Australia Incorporated. The study received ethics approval from the Health Research Ethics Committee (HREC), University of South Australia (Application ID: 0000034507).

Conflict of Interest: No conflict of interest has been declared by the authors.

\section{AUTHOR INFORMATION}

Dr Anne Hofmeyer is a tenured academic in the School of Nursing and Midwifery, Faculty of Health Sciences, University of South Australia, Australia. Visiting Professor of Anglia Ruskin University, UK.

E-mail: anne.hofmeyer@unisa.edu.au (Corresponding author)

Dr Luisa Toffoli is Program Coordinator: Bachelor of Nursing in the School of Nursing and Midwifery, Faculty of Health Sciences, University of South Australia, Australia.

Associate Professor Rachael Vernon is Associate Head of School: Academic in the School of Nursing and Midwifery, Faculty of Health Sciences, University of South Australia, Australia.

Professor Ruth Taylor is Pro Vice Chancellor and Dean, Faculty of Health, Social Care and Education at Anglia Ruskin University, Cambridge, United Kingdom.

Professor Dorrie Fontaine is Sadie Health Cabaniss Professor of Nursing and Dean at the University of Virginia, School of Nursing, Charlottesville, Virginia, United States of America.

Professor Hester C. Klopper, Vice-Rector (Deputy Vice-Chancellor) Strategic Initiatives and Internationalisation at Stellenbosch University, Cape Town, South Africa.

Associate Professor Siedine Knobloch Coetzee is at the School of Nursing Science, North-West University, Potchefstroom Campus, South Africa.

\section{REFERENCES}

Adam, D. \& Taylor, R. (2013). Compassionate care: Empowering students through nurse education. Nurse Education Today, 34(9), 1242-1245.

Adamson, L. \& Dewar, B. (2011). Compassion in the nursing curriculum: making it more explicit. Journal of Holistic Healthcare, 8(3), 42-45.

Adamson, L. \& Dewar, B. (2014). Compassionate care: Student nurses' learning through reflection and the use of story. Nurse Education in Practice, 15(3), 155-161.

Aigner, G. (2011). Leadership: Beyond Good Intentions. Allen \& Unwin New South Wales.

Aiken, L., Rafferty, A.M. \& Sermeus, W. (2014) Caring nurses hit by a quality storm. Nursing Standard, 28(35), $22-25$.

Angelou, M (2014). Maya Angelou Quotes: 15 of the best. http://www.theguardian.com/books/2014/may/28/maya-angelou-infifteen-quotes

Bartels, J.B. (2014). The Pause. Critical Care Nurse, 34(1), 74-75.

Bauer-Wu, S. \& Fontaine, D. (2015). Prioritizing Clinician Wellbeing: University of Virginia's Compassionate Care Initiative. Global Advances in Health and Medicine, 4(5), 16-22. 
Beaumont, E., Durkin, M., Hollins, C. \& Carson, J. (2016). Compassion for others, self-compassion, quality of life and mental well-being measures and their association with compassion fatigue and burnout in student midwives: A quantitative survey. Midwifery, http://dx.doi.org/10.1016/j.midw.2015.11.002

Benner, P. \& Wrubel, J. (1989). Primacy of Caring. Addison-Wesley, Menlo Park.

Berger, J., Polivka, B., Smoot, E. \& Owens, H. (2015). Compassion fatigue in pediatric nurses. Journal of Pediatric Nursing, 30(6), e11-e17.

Bevis, E.O. \& Watson, J. (1989). Toward a caring curriculum: A new pedagogy for nursing. National League for Nursing Press, New York

Borbasi, S. \& Jackson, D. (2016). Navigating the Maze of Research: Enhancing Nursing and Midwifery Practice, 4e Elsevier, Sydney, NSW.

Bramley, L. \& Matiti, M. (2014). How does it really feel to be in my shoes? Patients' experiences of compassion within nursing care and their perceptions of developing compassionate nurses. Journal of Clinical Nursing, 23(2), 790-799.

Braun, V. \& Clarke, V. (2013). Successful Qualitative Research. Sage Publications

Bray, L., O’Brien, M., Kirton, J., Zubairu, K. \& Christiansen, A. (2014). Role of professional education in developing compassionate practitioners. Nurse Education Today, 34(3), 480-486.

Campbell-Sills, L. \& Stein, M. (2007). Psychometric Analysis and Refinement of the Connor-Davidson Resilience Scale. Journal of Traumatic Stress, 20(6), 1019-1028.

Christiansen, A., O’Brien, M., Kirton, J., Zubairu, K. \& Bray, L. (2015). Delivering compassionate care: enablers and barriers. British Journal of Nursing, 24(16), 833-837.

Clark, C., Olender, L., Kenski, D. \& Cardoni, C. (2013). Exploring and Addressing Faculty-to-Faculty Incivility: A National Perspective and Literature Review. Journal of Nursing Education, 52(4), 1-8.

Clark, C. (2013). Creating \& Sustaining Civility in Nursing Education. Sigma Theta Tau International. Indianapolis, USA.

Coetzee, S. \& Klopper, H. (2010). Compassion fatigue within nursing practice: a concept analysis. Nursing and Health Studies, 12(2) 235-243.

Cornwell, J. \& Goodrich, J. (2009). Ensuring compassionate care in hospital. Nursing Times, 105(15), $14-16$.

Crawford, P., Brown, B., Kvangarsnes, M. \& Gilbert, P. (2014). The design of compassionate care. Journal of Clinical Nursing, 23(23-34), 3589-3599.

Cummings, G. (2012). Your leadership style: How are you working to achieve a preferred future? Journal of Clinical Nursing, 21(23-24), 3325-3327.

Cummings, J. \& Bennett, V. (2012). Compassion in practice: nursing, midwifery and care staff our vision and strategy. Retrieved from www.commissioningboard.nhs.uk.

Curtis, K. (2013). $21^{\text {st }}$ Century challenges faced by nursing faculty in educating for compassionate practice: Embodied interpretation of phenomenological data. Nurse Education Today, 33(7), 746-750.

Curtis, K., Horton, K. \& Smith, P. (2012). Student nurse socialisation in compassionate practice: a Grounded Theory study. Nurse Education Today, 32(7), 790-5.

Curtis, K. (2014). Learning the requirements for compassionate practice: student vulnerability and courage. Nursing Ethics, 21, $210-23$.

Curtis, K. (2015). Compassion is an essential component of good nursing care and can be conveyed through the smallest actions. Evidence Based Nursing, 18(3), 95.

Dalai Lama. (2003). Transforming the Mind: Teachings on Generating Compassion. Hammersmith, UK.

Darbyshire, P. \& McKenna, L. (2013). Nursing's crisis of care: What part does nursing education own? Nurse Education Today, 33(4), 305-307.

Davidson, P., Daly, J. \& Hill, M. (2013). Looking to the future with courage, commitment, competence and compassion. Journal of Clinical Nursing, 22(19-20), 2665-2667.

Dewar, B., Adamson, E., Smith, S., Surfleet, J. \& King, L. (2014). Clarifying misconceptions about compassionate care. Journal of Advanced Nursing, 70(8), 1738-1747.

Drennan, J. (2003). Cognitive interviewing. Journal of Advanced Nursing, 42(1), 57-63.

Drury, V., Craigie, M., Francis, K., Aoun, S. \& Hegney, D. (2014). Compassion satisfaction, compassion fatigue, anxiety, depression and stress in registered nurses in Australia: Phase 2 results. Journal of Nursing Management, 22(4), $519-531$.

Dunwoody, A. (2015). A Higher Standard. Da Capo Press, Philadelphia, PA. USA.

Fontaine, D. (2012). Compassionate Care \& Empathic Leadership Initiative. School of Nursing, University of Virginia, Virginia USA Retrieved from https://vimeo.com/54874862

Francis, R. (2013). Mid Staffordshire NHS Foundation Trust Inquiry. Final Report. Stationary Office, UK

Gaut, D. \& Leninger, M. (1991). Caring: The Compassionate Healer. New York: National League for Nursing Press.

Gilbert, P. (2005). Compassion and cruelty: A biopsychosocial approach. In: Gilbert P (ed.). Compassion: Conceptualisations, Research and Use in Psychotherapy. London: Routledge, pp. 9-74.

Glaser, J. (1994). Three Realms of Ethics. Kansas City, MO: Sheed \& Ward.

Goetz, J., Keltner, D. \& Simon-Thomas, E. (2010). Compassion: an evolutionary analysis and empirical review. Psychological Bulletin, 136, 351-374. 
Guba, E. \& Lincoln, Y. (1994). Competing paradigms in qualitative research. In N.K. Denzin \& Y.S. Lincoln (Eds.), Handbook of qualitative research. 105-117 Sage.

Hayter, M. (2013). UK Francis Report: Key messages. Journal of Advanced Nursing, 69(8), e1-e2.

Heffernan, M., Quinn Griffin, M.T., McNulty, S.R. \& Fitzpatrick, J.J. (2010). Self-compassion and emotional intelligence in nurses. International Journal of Nursing Practice, 16(4) 366-373.

Hofmeyer, A., Sheingold, B. \& Taylor, R. (2015). Do you understand what I mean? Cognitive interviewing to develop valid reliable study instruments and dissemination products. Journal of International Education Research, 11(4), 261-268.

Hofmeyer, A. (2013). How can a social capital framework guide managers to develop positive nurse relationships and patient outcomes? Journal of Nursing Management, 21(5), 782-789.

Hofmeyer, A., Sheingold, B., Klopper, H. \& Warland, J. (2015). Leadership in learning and teaching in higher education: Perspectives of academics in non-formal leadership roles. Contemporary Issues in Education Research, 8(3), 181-192.

Holloway, S. (2013). Resilience in Mind: Early action for mental health. Retrieved from http://www.communitylinks.org/linksuk/?p=3910

Horsburgh, D. \& Ross, J. (2013). Care and compassion: the experience of newly qualified staff nurses. Journal of Clinical Nursing, 22(7-8), 1124-1132.

Hunsaker, S., Chen, H., Maughan, D. \& Heaston, S. (2015). Factors that influence the development of compassion fatigue, burnout, and compassion satisfaction in emergency department nurses. Journal of Nursing Scholarship, 47(2), $186-194$.

Izhaki, M., Treacy, M., Phaladze, N., Rumeu, C., Vernon, R., Marshall, B., Fealy, G., Ehrenfeld, M., Larkin, P., McNamara, M., Dignam, D., Rollins-Ganz, N. \& Nelson, J. (2015). A five country partnership to measure perception of nursing staffs' caring for self, burnout, and compassion fatigue. Interdisciplinary Journal of Partnership Studies, 2(1), 1-20. http://pubs.lib.umn.edu/ijps/

Jackson, D., Firtko, A. Edenborough, M. (2007). Personal resilience as a strategy for surviving and thriving in the face of workplace adversity: Literature review. Journal of Advanced Nursing, 60(1), 1-9.

Joinson, C. (1992). Coping with Compassion Fatigue. Nursing, 22(4), 116-122.

Kenny, G. (2016). Compassion for simulation. Nurse Education in Practice, 16(1), 160-162.

Klopper, H.C. (2015). Opening Plenary Address. Sigma Theta Tau International $26^{\text {th }}$ International Research Congress, San Juan, Puerto Rico, 23-27 July

Lamothe, M., Boujut, E., Zenasni, F. \& Sultan, S. (2014). To be or not to be empathic: the combined role of empathic concern and perspective taking in understanding burnout in general practice. BMC Family Practice, 15(15).

Leary, M., Tate, E., Adams, C., Allen, A. \& Hancock, J. (2007). Self-compassion and reactions to unpleasant self-relevant events: The implications of treating oneself kindly. Personality and Individual Processes, 92(5), 887-904.

Lee, Youngjin. \& Seomun, G. (2016). Development and validation of an instrument to measure nurses' compassion competence. Applied Nursing Research, 30(2), 76-82.

Leininger, M. \& Watson J. (1990). The caring imperative in education. National League for Nursing Press, New York.

Lincoln, Y. \& Guba, E. (1985). Naturalistic Inquiry. Beverley Hills: Sage Publications

Lown, B., Rosen, J. \& Marttila, J. (2011). An Agenda for improving compassionate care: A survey shows about half of patients say such care is missing. Health Affairs, 30(9), 1772-1778.

Lown, B. (2014). Seven Guiding Commitments: Making the U.S. healthcare system more compassionate. Journal of Patient Experience, 2(1), 6-15.

Maben, J., Sweeney, K. \& Cornwell, J. (2010). In praise of compassion. Journal of Nursing Research, 15(9), 9-13.

McAllister, M. \& McKinnon, J. (2009). The importance of teaching and learning resilience in the health disciplines: a critical review of the literature. Nurse Education Today 29(4), 371-379.

McCaffrey, G. \& McConnell, S. (2015). Compassion: a critical review of the peer-reviewed nursing literature. Journal of Clinical Nursing, 24(19-20), 3006-3015.

McPherson, S., Hiskey, S. \& Alderson, Z. (2016). Distress in working on dementia wards - A threat to compassionate care: A grounded theory study. International Journal of Nursing Studies, 53, 95-104

McDonald, G., Jackson, D., Vickers, M. \& Wilkes, L. (2016). Surviving workplace adversity: a qualitative study of nurses and midwives and their strategies to increase personal resilience. Journal of Nursing Management, 24(1), 123-131.

Mills, J., Wand, T. \& Fraser, J. (2015). On self-compassion and self-care in nursing: Selfish or essential for compassionate care? International Journal of Nursing Studies, 52(4), 791-793.

Neff, K. (2003a). Development and validation of a scale to measure self-compassion. Self and Identity, 2(3), 223 -250.

Neff, K. (2003b). Self-compassion: An alternative conceptualization of a healthy attitude toward oneself. Self and Identity, 2(2), $85-101$.

NHS Confederation (2008). Compassion in healthcare: Missing dimension of healthcare reform? Paper 2

Nightingale, F. [First published 1859]. Notes on Nursing: What it is and what it is not. Glasgow \& London: Blackie \& Son Ltd. ISBN 0-216-89974-5.

NMSF (2013-15). Caring with Kindness: Nursing and Midwifery Professional Practice Framework SA Health, Dept. for Health and Ageing Government of South Australia Retrieved from http://www.sahealth.sa.gov.au/

Nussbaum, M. (2003). Compassion and terror. Daedalus 132, 10e26. 
Papadopoulos, I. \& Ali, S. (2016). Measuring compassion in nurses and other healthcare professionals: An integrated review. Nurse Education in Practice, 16(1), 133-139.

Patton, M.Q. (1990). Qualitative evaluation and research methods. 2e Newbury Park, CA: Sage Publ.

Pitt, V., Powis, D., Levitt-Jones, T. \& Hunter, S. (2014). Nursing students' personal qualities: A descriptive study. Nurse Education Today, 34(9), 1196-1200.

Polit, D. \& Beck, C. (2011). Nursing Research: Principles and Methods (9th ed.). Philadelphia, Lippincott.

Rafferty, A.M. (2011). We can read Nightingale as a credo for compassion today. Nursing Times Ethical and Compassionate Nursing Supplement, 24 August 3.

Reyes, D. (2012). Self-Compassion: A Concept Analysis. Journal of Holistic Nursing, 30(2), 81-89.

Richardson, C., Percy, M. \& Hughes, J. (2015). Nursing therapeutics: Teaching student nurses care, compassion and empathy. Nurse Education Today, 35(5), e1- e5.

Ruona, W. (2005). Analyzing qualitative data. In R. Swanson \& E. Holton (Eds.), Research in Organisations. pp. 233-263. San Francisco, CA: Berrett-Koehler.

Sandelowski, M. (2010). What's in a name? Research in Nursing \& Health, 33(1), 77-84.

Schantz, M. (2007). Compassion: A Concept Analysis. Nursing Forum, 42(2), 48-55.

Schout, G., de Jong, G. \& Zeelen, J. (2009). Establish contact and gaining trust: An exploratory study of care avoidance. Journal of Advanced Nursing, 66(2), 324-333.

Senyuva, E., Kaya, H., Isik, B. \& Bodur, G. (2013). Relationship between self-compassion and emotional intelligence in nursing students. International Journal of Nursing Practice, 20(6), 588- 596.

Seppälä E. (2015). Why compassion is a better managerial tactic than toughness. Harvard Business Review, Retrieved from https://hbr.org/2015/why-compasion-is-a-better-managerial-tactic-then-toughness

Seppala, E., Hutcherson, C., Nguyen, D., Doty, J. \& Gross, J. (2014). Loving-kindness meditation: a tool to improve healthcare provider compassion, resilience and patient care'. Journal of Compassionate Health Care 1:5 DOI 10.1186/s40639-014$0005-9$

Sieg, D. (2015). 7 habits of highly resilient nurses. Reflections on Nursing Leadership, 41(1), 1-3.

Sinclair, S., McClement, S., Raffin-Bouchal S., Hack T., Hagen N., McConnell, S. Chochinov H. (2016a) Compassion in Healthcare: Empirical Model. Journal of Pain Symptom Management, 51(2), 193-203.

Sinclair, S., Norris, J., McConnell, S., Max Chochinov, M., Hack, T., Hagen, N., McClement, S. \& Bouchal, S. (2016b). Compassion: a scoping review of the healthcare literature. BMC Palliative Care, 15(6) DOI 10.1186/s12904-016-0080-0

van der Cingel, M. (2014). Compassion: The missing link in quality of care. Nurse Education Today, 34(9), 1253-1257.

von Dietze, E. \& Orb, A. (2000). Compassionate care: a moral dimension of nursing. Nursing Inquiry 7(3), 166-174.

Weinstein, M. (2014). B is for Balance: 12 Steps Toward a More Balanced Life at Home and at Work. 2e Sigma Theta Tau International. Indianapolis, USA.

West, M. (2014). Culture in Organizations. Royal College of Nursing (RCN) International Nursing Research Conference, Glasgow, Scotland.

Yoder, E. (2010). Compassion fatigue in nurses. Applied Nursing Research, 23(4), 191-197. 


\section{APPENDIX 1: STUDY PROTOCOL}

\section{PRE-INTERVENTION QUALITATIVE QUESTIONS}

We are interested to know how undergraduate nursing students understand the topic of compassion in nursing. We are inviting you to respond to five open-ended questions in Part A. Please type your responses into the text box under each question. Part B consists of seven demographic questions. These questions should take no longer than ten minutes to complete. Your ideas are very important to us. By submitting your responses, you are consenting to participate in the study. All responses are confidential, and participation is voluntary. All responses are anonymous and will only be viewed by the researchers. Findings will be used to improve the teaching of this topic to future undergraduate nursing students. This study has received ethical approval from XX Review Board. Thank you for taking part in our study.

Part A

1. What does compassion mean to you?

2. What does practicing compassion mean to you?

3. Can you give an example of you (or someone else) being compassionate towards a client, resident or patient?

4. Why do you think this is a good example?

5. What six words summarize this topic for you?

\section{Part B}

1. Are you: $17-21$ years; $22-29$ years; $30-39$ years; 40 - 49 years; $50-59$ years; other - please specify.

2. What is your gender: female; male

3. Are you enrolled as an international student: yes; no

4. What language do you speak most commonly at home?

5. In what country were you born?

6. If you were not born in this country, in what year did you arrive?

7. What is your highest qualification?

\section{KNOWLEDGE INTERVENTION: COMPASSION MODULE}

The importance of providing compassionate care to patients and families is well established. 'Cultivating a resilient healthcare workforce committed to high quality, compassionate, relationship based care' (Bauer-Wu \& Fontaine, 2015, p.16) is championed globally, and is the cornerstone of many health policies (NHS, 2008; NMSF, 2013-15). Caring and being compassionate is the essence and moral foundation of nursing practice and education (Bevis \& Watson, 1989; Benner \& Wrubel, 1989; Leininger \& Watson, 1990). The practice of compassion is as vital as clinical competence in quality healthcare (Davidson et al., 2013; van der Cingel, 2014). Compassion is defined as 'a virtuous response that seeks to address the suffering and needs of a person through relational understanding and action' (Sinclair et al., 2016a, p.193). The intention to act to relieve the suffering of others distinguishes compassion from other virtuous qualities (Maben et al., 2010; Papadopoulos \& Ali, 2016; Schantz, 2007). In this Compassion Module, eight questions are posed and evidence-based responses are provided. Reflective questions to enhance your selfawareness and key readings conclude each section.

\section{How can I understand compassion?}

'Compassion is how care is given through relationships based on empathy, respect and dignity - it can also be described as intelligent kindness and is central to how people perceive their care' (Cummings \& Bennett, 2012). To be cared for with compassion is an expectation of patients and their families. The smallest things matter and can make a big difference to patients (Adamson \& Dewar, 2011). This is why communication is crucial, because what matters to patients may not be the same priority for the nurse (Adamson \& Dewar, 2011). Knowing what matters to patients to 
preserve their dignity helps nurses provide ethical and professional care that is perceived as high-quality and compassionate by patients and families.

Compassionate care matters to patients and is central to the idea of what it means to be a nurse (Bray et al., 2014). Notably, patients and their families say the manner in which they are listened to and cared for with compassion matters just as much as the quality of health care itself (Bray et al., 2014; Lown et al., 2011; Schout et al., 2009). Compassionate care addresses the patient's core need for 'connection and relationships and is based on attentive listening and a desire to understand the patient's context and perspective' (Lown et al., 2011). Its presence enhances effectiveness and patient-centred care (Lown, 2014). Compassion can be felt through a quiet attentive presence, respect, and sensitivity to what is said and not said. It can 'ameliorate concerns, distress or suffering' (Lown, 2014, p. 6). But importantly, compassion is not only essential for the high quality care of patients, but also for reducing burnout and improving health outcomes, well-being, and resilience in clinicians themselves (Bauer-Wu \& Fontaine, 2015; Seppala et al 2014; Lamothe et al 2014).

\section{Reflective Questions:}

- What are your beliefs and values about being compassionate?

- What does it mean to you to learn that compassion is important for reducing burnout and improving wellbeing and resilience in clinicians?

Key Readings:

Adamson, L. \& Dewar, B. (2011). Compassion in the nursing curriculum: making it more explicit. Journal of Holistic Healthcare, 8(3), 42-45.

Bray, L., O'Brien, M., Kirton, J., Zubairu, K. \& Christiansen, A. (2014). The role of professional education in developing compassionate practitioners. Nurse Education Today, 34(3), 480-486.

\section{Why does compassion in healthcare matter and what are the barriers?}

There is an increasing concern that, despite significant resources devoted to healthcare and a focus on quality improvement and evidence-based practice, systems are still failing to meet the holistic care needs and compassionate care expectations of patients and their families (Bray et al., 2014; Lown, 2014; Lown et al., 2011). Reports indicate that communication and compassion are not consistent features in healthcare systems that are under increasing strain (Aiken et al., 2014). International research has linked poor outcomes for patients with austerity measures and the perceived lack of compassion in healthcare (Bray et al., 2014). Healthcare professionals face numerous challenges (barriers) in the system such as excessive workloads and incivility that hinder their ability to provide effective, compassionate care (Lown, 2014; Clark et al., 2013).

So why does compassion in healthcare organisations matter? Hundreds of older frail adults died at two hospitals in the Mid Staffordshire Foundation NHS Trust between 2005 and 2009. The Francis Review identified factors such as: system failure to provide fundamental nursing care, abysmal standards of care, inadequate hydration and nutrition, lack of patient privacy and dignity, professional conflict, intimidating and financially driven management culture, and a widespread lack of compassion that persisted for many years (Hayter, 2013, p. e1). The absence of compassion was devastating for both patients and healthcare providers and in turn, eroded community trust in healthcare systems. Compassion is thoughtfulness and recognition of the human needs of others (Francis, 2013). Some are calling for these debates to move on. But lessons must be learned from the past. Barriers that hinder the provision of high-quality, compassionate care can be identified at the individual and organisational levels (Christiansen et al., 2015). Standards of healthcare are still lacking in systems and cultures, despite the availability of abundant evidence. So what needs to be transformed?

Rafferty (2011, p. 3) reminds us, 'Nightingale recognised that systems are needed to foster and institutionalise compassion, and that small details mattered'. Leadership by all nurses is urgently needed to improve workplace cultures, quality care, and sustainable change. Culture can be understood as 'the way we do things around here' (West, 
2014). It is timely for all nurses to reflect on the quality of care they are providing for their patients and the cultural norms and networks that shape their workplace environments. Are the norms and networks inclusive, respectful and cooperative (i.e., high in social capital) or is incivility and exclusion commonplace? Evidence from previous literature confirms that poor care is associated with poor care environments (Aiken et al., 2014). Moreover, 'ongoing high stress takes a toll on clinicians and interferes with the quality of their patients care'. Thus, fostering clinician well-being and resilience needs to be a priority (Bauer-Wu \& Fontaine, 2015, p. 16). It is timely for nurses to act collaboratively to implement strategies to improve workplace cultures, do the right thing (moral leadership), and strengthen health care environments (Hofmeyer 2013). Practising compassion is foundational in person-centred care and enhances job satisfaction for nurses.

Increasing attention has been given to teaching compassion in nursing programs to improve compassionate practice in clinical settings (see, for example: Bauer-Wu \& Fontaine, 2015; Adam \& Taylor, 2013; Adamson \& Dewar, 2014; Adamson \& Dewar, 2011; Dewar et al., 2014; Cummings \& Bennett, 2012; Bray et al., 2014).

\section{Reflective Questions:}

- Why do you think compassion in health care matters?

- What do you think are some of the barriers to providing compassionate care in healthcare workplaces?

- How do you think compassion could be taught in the BN program?

Key Readings:

Adam, D. \& Taylor, R. (2013). Compassionate care: Empowering students through nurse education. Nursing Education Today, 34(9), 1242-1245

Lown, B. (2014). Seven Guiding Commitments: Making the U.S. healthcare system more compassionate. Journal of Patient Experience, 2(1), 6-15.

Christiansen, A., O’Brien, M., Kirton, J., Zubairu, K. \& Bray, L. (2015). Delivering compassionate care: the enablers and barriers. British Journal of Nursing, 24(16), 833-837.

\section{What does 'practicing compassion' mean?}

There is irrefutable evidence about the benefits of nurses practicing compassion toward others and the negative impact to nurses and their patients when compassion is lacking in healthcare, organizational systems and workplace cultures (Lown et al 2011, Aiken et al 2014). Further, Lown and colleagues (2011) argue that 'medical care without compassion cannot be truly patient-centered'. Likewise, nursing care without compassion cannot be truly patient-centered. So while it is clear that compassion matters, what is less clear to many students is what 'practicing compassion' actually means. These insights are essential to increase understanding and provide evidence about how nursing students can strengthen their practice of 'compassion as a core professional value' (Bray et al 2014, p. 480).

We can learn from others. So you are invited to watch an 18 minute video about the Compassionate Care Initiative (CCI) that was developed by nurse leaders at the Faculty of Nursing, University of Virginia, USA (Fontaine, 2012). The CCI is guided by 4 central concepts and values: resilience, mindfulness, interprofessional collaboration, and healthy work environment (Bauer-Wu \& Fontaine, 2015, p. 18). In the video, nurses, physicians, administrators, professors and students explain what 'practicing compassion' means for them in their everyday lives. They talk about practicing compassion toward themselves, colleagues and patients, and how they strengthen their own resilience through an ongoing mindful process. In addition, the short reading by Bartels (2014) provides a potent example of practicing compassion in the clinical setting and the perceived benefits for the healthcare team. Please click on this link to play the video. Alternatively, copy the address below into google to access the video:

UVa School of Nursing's Compassionate Care \& Empathic ... Retrieved from https://vimeo.com/54874862 
Reflective Questions:

- What does practicing compassion mean to you?

- Cite an example of you (or someone else) practicing compassion toward a patient.

- Do you think it is more than showing kindness to others? Why?

Key Readings:

Bartels, J.B. (2014). The Pause. Critical Care Nurse, 34(1), 74-75.

Bauer-Wu, S. \& Fontaine, D. (2015). Prioritizing Clinician Wellbeing: University of Virginia's Compassionate Care Initiative. Global Advances in Health and Medicine, 4(5), 16-22.

\section{What does 'leading with compassion' mean?}

Dunwoody (2015, p. 255) reminds us to: 'never walk by a mistake, or you just set a new lower standard'. This means you need to try to improve the situation for every patient and colleague. Maybe you have thought that only nurses in formal leadership management positions such as nurse managers can lead and change situations. What we are saying is that all nurses can chose to lead with compassion, and therefore influence what happens around them. So how could you respond when you see a colleague under-performing or making an error in the healthcare workplace? How could you influence that outcome for the better? Leading with compassion is about speaking up in those situations and exerting your power and influence by setting the tone for doing what is right, even in difficult circumstances.

Compassion means to be kind to others (Sieg, 2015). But don't confuse that idea with not providing truthful, timely feedback in a respectful manner. A common approach to dealing with mistakes is punitive which you may have experienced. But that approach weakens trust and loyalty, Seppälä (2015) suggests we respond with 'compassion and curiosity' and therefore, seek to understand the other person's point of view. This requires us to 'suspend judgement' as we 'listen' and try to understand what led to the error. This approach does not mean we sanction poor performance. Rather, our compassionate response as a compassionate leader strengthens our relationships with others and influences the culture in a productive way to improve service and client outcomes and satisfaction. The challenge is to pause for a moment to manage our emotions before we respond. This is known as exercising emotional intelligence. Thus, Seppälä (2015) explains, 'by choosing a compassionate response when they have made a mistake, they are not destroyed, they have learned a lesson, and they want to improve for you because you've been kind to them'.

As Cummings (2012, p. 3325) clarifies, 'leadership is being able to see the present for what it really is, see the future for what it could be, and then, take action to close the gap'. Management is about reducing risk; keeping things the same, standardized. Leadership is about taking calculated and planned risk in order to achieve a preferred future. Leadership is action, not position (Cummings 2012, p. 3325). Leadership is the ability to influence direction. This means you can be a leader and work toward the future you want as a new registered nurse. 'A leader is someone that people jump ship to follow' (Hofmeyer et al., 2015, p.185). So leadership is about making ourselves useful to our organisation, our community, our family, and our world (Aigner, 2011, p. x).

\section{Reflective Questions:}

- Analyse why you think is it important to be compassionate toward colleagues?

- Can you think of an example of a colleague being compassionate toward you?

- How did it make you feel?

- Can you think of an example of a colleague NOT being compassionate toward you?

- How did it make you feel?

Key Readings:

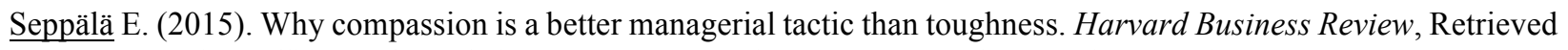
from https://hbr.org/2015/why-compasion-is-a-better-managerial-tactic-then-toughness

Copyright by author(s); $\underline{\text { CC-BY }}$ 


\section{How can I understand self-compassion?}

The majority of research studies have focused on how individual nurses can provide compassionate care for patients and their families. But the key message is that self-compassion must exist before individuals can practice compassion toward others. However, there is scant research on this topic (Mills et al., 2015). When we feel exhausted, tired and depleted, we have little energy to care for others in a meaningful way. Mills and colleagues (2015) identified only three studies that investigated nurses' self-compassion and two of those studies showed a positive correlation with emotional intelligence (Heffernan, 2010; Senyuva, 2013).

The Dalai Lama (2003, p. 125) explains the importance of self-compassion and its relationship our capacity to practice compassion toward colleagues and patients. He says:

For someone to develop genuine compassion towards others, first he or she must have a basis upon which to cultivate compassion, and that basis is the ability to connect to one's own feelings and to care for one's own welfare. . Caring for others requires caring for oneself.

So what does self-compassion and the practice of self-care really mean? Is it indulgent, is it being selfish? (Mills et al., 2015) The answer is no. Self-care and kindness is essential to our well-being and the foundation for practising compassion toward our colleagues and patients. We can understand self-compassion 'as a pattern of increased selfcare capacity, compassion for others, and increased relatedness, autonomy and sense of self' (Reyes, 2012, p 81). A major contribution to research in this area was made by Dr. Kristin Neff. She is the leading global researcher in selfcompassion research and explains that compassion for self is equally important to having compassion for others and has many important psychological benefits. Self-compassion is about having feelings of kindness for oneself, as one would have for others (Neff, 2003a). But more often we are very hard on ourselves. Neff (2003a) developed a 26-item Self-Compassion Scale (SCS) to measure self-compassion (six subscales/components). We are using the questions in this SCS survey to explain and help you understand what self-compassion actually means in your life. The three components of self-compassion are:

Self-Kindness:

1. I try to be loving towards myself when I'm feeling emotional pain;

2. When I'm going through a hard time, I give myself the caring and tenderness I need;

3. I'm kind to myself when I'm experiencing suffering;

4. I'm tolerant of my own flaws and inadequacies;

5. I try to be understanding and patient towards those aspects of my personality I don't like.

Common Humanity:

1. When things are going badly for me, I see the difficulties as part of life that everyone goes through;

2. When I'm down and out, I remind myself that there are lots of other people in the world feeling like I am;

3. When I feel inadequate in some way, I try to remind myself that feelings of inadequacy are shared by most people;

4. I try to see my failings as part of the human condition.

Mindfulness:

1. When something upsets me I try to keep my emotions in balance;

2. When something painful happens I try to take a balanced view of the situation;

3. When I fail at something important to me I try to keep things in perspective;

4. When I'm feeling down I try to approach my feelings with curiosity and openness. 
The three components that are barriers to self-compassion are:

Self-Judgment:

1. I'm disapproving and judgmental about my own flaws and inadequacies.

2. When times are really difficult, I tend to be tough on myself.

3. I'm intolerant and impatient towards those aspects of my personality I don't like.

4. When I see aspects of myself that I don't like, I get down on myself.

5. I can be a bit cold-hearted towards myself when I'm experiencing suffering.

Isolation:

1. When I think about my inadequacies, it tends to make me feel more separate and cut off from the rest of the world.

2. When I'm feeling down, I tend to feel like most other people are happier than I am.

3. When I'm really struggling, I tend to feel like other people must be having an easier time of it.

4. When I fail at something that's important to me, I tend to feel alone in my failure.

Over-Identification:

1. When I'm feeling down I tend to obsess and fixate on everything that's wrong.

2. When I fail at something important to me I become consumed by feelings of inadequacy.

3. When something upsets me I get carried away with my feelings.

4. When something painful happens I tend to blow the incident out of proportion.

It is important to understand that others have similar experiences to ourselves (Neff, 2003b).You can read about Dr Kristin Neff's research and access the SCS at her website: http://self-compassion.org/ Self-compassion is about selfcare during difficult times that can build resilience to cope in demanding work environments and circumstances. Anger, judgment, impatience and resentment can be self-destructive and lock us into negative emotions. Self-care is about knowing when to 'let go' and move on (Sieg 2015). Self-compassion is about expressing understanding, kindness and concern to yourself in the same way you would to a friend (Leary et al., 2007).

Reflective Questions:

- How could you develop a plan to practice self-care and kindness?

- Interestingly, few studies have focused on nurses' self-compassion. How would you write a research question to generate new knowledge about that topic?

Key Readings:

Neff, K. (2003a). Development and validation of a scale to measure self-compassion. Self and Identity, 2(3), 223 250.

Mills, J., Wand, T. \& Fraser, J. (2015). On self-compassion and self-care in nursing: Selfish or essential for compassionate care? International Journal of Nursing Studies, 52(4), 791-793.

\section{What is compassion fatigue and how can I build my resilience?}

Evidence confirms the benefits of compassion for quality patient outcomes, for clinicians, healthcare organisations and society. However, barriers such as demanding working conditions, heavy workloads, staff shortages, low team morale, indifferent management, inadequate training, and limited resources continue to characterise healthcare environments that in turn, compromise the delivery of quality care and foster staff stress (Aiken et al., 2014). Furthermore, 'incivility in the healthcare workplace is pervasive: stressed professionals treat their colleagues with disrespect, thereby perpetuating the stressful work environment' (Bauer-Wu \& Fontaine, 2015, p 17). High stress 
compromises clinicians' clear thinking and clinical decision-making, thus increasing the risk of errors and threatening patient safety (Bauer-Wu \& Fontaine, 2015, p 17).

These organisational problems can result in compassion fatigue for individuals (Coetzee \& Klopper, 2010). Compassion fatigue was first used by Joinson to describe nurses who had lost their 'ability to nurture' (1992 p. 119). However, fatigue is more than feeling tired. Weinstein (2014) explained that 'fatigue is mental or physical exhaustion that stops a person from being able to function normally'. Compassion fatigue (emotional exhaustion, sense of burnout, lack of empathy and connection) can negatively affect professional and personal relationships, and work performance (Hunsaker et al., 2015; Berger et al., 2015). Evidence has shown that compassion fatigue limits nurses' ability to care for patients (Izhaki et al., 2015). Clinicians experiencing burnout are more likely to depersonalize patients such as referring to them as their disease (Bauer-Wu \& Fontaine, 2015).

Compassion fatigue can be understood as nurses 'losing their nurturing ability toward patients' (Coetzee \& Klopper, 2010) toward colleagues, and toward themselves. Evidence suggests that many nurses have diminished resilience. Therefore, the ability of exhausted nurses to reflect on their practice to provide compassionate care for patients and work as an effective team member is under threat (Aiken et al., 2014; Dewar et al., 2014). More than 40 percent of nurses working in hospital environments report physical, emotional and/or mental exhaustion (Sieg, 2015). Demanding workplaces, exhaustion and fatigue can lead to burnout which is the antithesis of feeling energised, focused and committed to quality caring. Some wrongly believe it is unprofessional or a sign of weakness to disclose their compassion fatigue. But their ability to work is compromised. The progression of compassion fatigue in nurses leads to increased risk in quality and safety in healthcare organisations and cultures. Managers need to strengthen organisational cultures and address austerity problems. In addition, professional education to empower nurses is essential and employee assistance programs must be readily available (Coetzee \& Klopper, 2010).

Learning to flourish in demanding contemporary healthcare environments is related to fostering personal resilience. Notably, 'resilience is the capacity to accurately perceive and respond well to stressful situations. It is demonstrated not only in times of crisis, but every day by showing up and doing our work. With the uncertainty, transition, and reorganisation associated with health care, resilience is more important than ever if today's nurse is going to thrive' (Sieg, 2015, p.1). If the demands persist, compassion fatigue becomes a reality (Coetzee \& Klopper, 2010). Ultimately, patient care is compromised. What items give you comfort and foster positive feelings and resilience? The items might be inexpensive but carry significance and remind you of precious people, holidays, and memories that evoke happiness and hope (Sieg, 2015). Caring for others has a cost.

\section{Reflective Questions:}

- Examine how you deal with fatigue, exhaustion or feeling overwhelmed.

- Identify strategies (habits) could you use to foster positive feelings and optimism.

- What strategies could you use to establish boundaries to avoid compassion fatigue?

Key Readings:

Coetzee, S. \& Klopper, H. (2010). Compassion fatigue within nursing practice: a concept analysis. Nursing and Health Studies, 12(2), 235-243.

Aiken, L., Rafferty, AM. \& Sermeus, W. (2014). Caring nurses hit by a quality storm. Nursing Standard, 28(35), 2225 .

\section{How does self-compassion and self-care build my resilience?}

Healthcare environments are challenging, but resilient nurses understand that taking care of others also means taking care of themselves. Resilience refers to a person's ability to thrive, continue to be positive, and function despite difficult circumstances and adversity (Campbell-Sills \& Stein, 2007). Individuals are not born with levels of resilience. Holloway (2013) describes how resilience is not an inherent quality ... and is not simply an ability to 'bounce back'. Rather, it is a personal capacity that can be fostered with particular resources. Resilience is being able to manage 
challenging circumstances whilst maintaining positive mental health. The 'fuel' to build our resilience is comes through our self-care, our relationships, networks and communities. Socially isolated individuals have a limited ability to access to the necessary resources derived through networks to foster their well-being and resilience.

So how can you build your resilience to remain positive and flourish under pressure? What can you do to foster the necessary interpersonal skills to build effective relationships, foster hope, and practice self-compassion? Jackson et al (2007 p. 1) suggests: 'building positive and nurturing professional relationships; maintaining positivity; developing emotional insight; achieving life balance and spirituality; and becoming more reflective'. Likewise, Holloway (2013) identified three interconnected elements that are key to building resilience in individuals and communities: (1) promoting well-being; (2) building social capital \& networks; and (3) learning more about mood and mind.

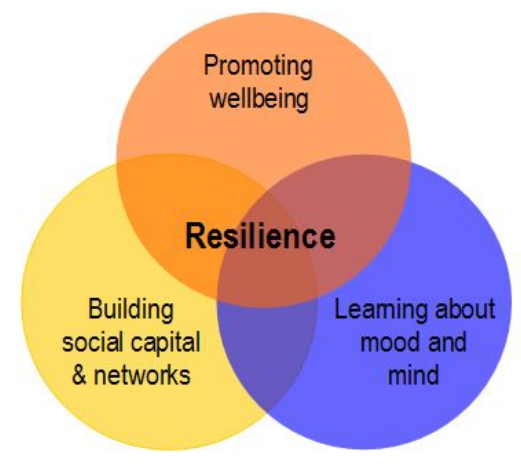

Strategies to build your resilience:

\section{(1) Promoting Well-being:}

- Build your physical resilience through nutrition, sleep, exercise. Go for a walk;

- Take time to relax;

- Have a purpose, set your goals, follow your dreams and work steadily toward success.

\section{(2) Building Social Capital \& Networks:}

- Capital is a resource with productive capacity (e.g., money);

- Foster your social resources, connections and networks;

- Nurture a diverse range of friendships and positive professional relationships so you have trusted people (peers, mentors) to connect with for encouragement and feedback;

- Be a trusted friend!

- Have a confidant who can help you find your inner strength.

(3) Learning about Mood and Mind:

- Gratitude is seeing and appreciating the blessings in our life (Sieg, 2015);

- Practice gratitude to enhance positive feelings and calm emotions;

- Mindfulness (focus on the present) and meditation can enhance well-being and resilience;

- Trust your intuition (instinct);

- Know when to let go and walk away;

- Reach out when you feel negative emotions building up;

- Develop your emotional intelligence to strengthen personal resilience;

- Build your self-reflection and insight - be honest with yourself;

- Practice self-compassion rather than self-criticism. 
McDonald and colleagues (2016, p. 129) remind us that all nurses share a responsibility to ensure they and others can practice in safe contemporary healthcare work environments: "Nursing management must ensure new graduates and early career nurses and midwives are aware of the potential effects of workplace adversity on their health and wellbeing. Nursing graduates may be forewarned of the potential dangers of professional burnout and may have practised self-reflection techniques; they should also understand how developing personal resilience could assist them to meet the demands of their careers." Moreover, Drury et al., (2014) have shown that resilient nurses are more likely to stay in the nursing workforce and importantly, the care they provide is higher-quality (p. 528). To care for others we must firstly care for ourselves.

\section{Reflective Questions:}

- What self-care practice can you start now to build your resilience?

- Identify what creates hope for you?

- What sustains your resilience?

- Identify a situation when you felt emotionally depleted and unable to care for patients with the energy and compassion that correspond to your values. Analyse what happened. What further knowledge and skills do you need to be more effective in delivering compassionate care? (Adam \& Taylor, 2014, p. 1243)

Key Readings:

McDonald G, Jackson D, Vickers M.H. \& Wilkes L, 2015, 'Surviving workplace adversity: a qualitative study of nurses and midwives and their strategies to increase personal resilience', Journal of Nursing Management 24(1), 123-131.

Clark, C., Olender, L., Kenski, D. \& Cardoni, C. (2013). Exploring and Addressing Faculty-to-Faculty Incivility: A National Perspective and Literature Review. Journal of Nursing Education, 52(4), 1-8.

\section{What are the key messages?}

Compassion is motivated by the intent to improve lives, including our own (Aigner, 2011). Compassion is an ethical activity, as something we do. At an individual level, compassion arises when there is self-compassion, reflection, respectful communication and behaviours, and resilience. At an organisational level - the practice of compassion arises when there are engaged and resilient individuals collaborating in respectful and inclusive cultures, supported by empathic leadership, effective structures and adequate resources. At a societal level - a compassionate society is civil, fair, inclusive, committed to the common good, and socially responsible (Glaser, 1994). So how can I practice compassion through my communication to be person-centred and foster therapeutic relationships? Adamson \& Dewar (2011, p. 44) ask the patient 'what is important to you right now?' Don't assume you know what a person needs. When was the last time you asked someone how they were - and then really listened to their response? Consider:

\begin{tabular}{l|l|l}
\hline \multicolumn{1}{c}{ Query } & \multicolumn{1}{c}{ Analysis } & \multicolumn{1}{c}{ Person may feel, think or say } \\
\hline How are you? & $\begin{array}{l}\text { An every-day question, but may seem uncaring and } \\
\text { insensitive }\end{array}$ & $\begin{array}{l}\text { What a thoughtless question, how do you think I } \\
\text { am. I am waiting for my test results and feel } \\
\text { anxious and scared - and you ask me how I am! }\end{array}$ \\
\hline $\begin{array}{l}\text { How are you } \\
\text { today? }\end{array}$ & $\begin{array}{l}\text { Question acknowledges things could be difficult for } \\
\text { the person, i.e., poor test results, life circumstances or } \\
\text { upsetting other issues. }\end{array}$ & $\begin{array}{l}\text { The person may feel that you are present with them } \\
\text { and trust you. }\end{array}$ \\
$\begin{array}{l}\text { BUT the word today focuses your query in their } \\
\text { present moment. }\end{array}$ & $\begin{array}{l}\text { They may feel safe to say how they really feel } \\
\text { today. They may feel a little better today - even } \\
\text { though their situation is overwhelming. }\end{array}$ \\
\hline $\begin{array}{l}\text { How are you in } \\
\text { yourself? }\end{array}$ & $\begin{array}{l}\text { Question acknowledges things could be difficult for } \\
\text { the person regarding their condition, test results, or } \\
\text { life circumstances. But the words in yourself focuses } \\
\text { the query on the person - their emotion, feelings, } \\
\text { spirit, hope. }\end{array}$ & $\begin{array}{l}\text { The person may feel they can share how they feel } \\
\text { about their situation, that you are non-judgemental, } \\
\text { and don't expect them to be brave. } \\
\text { The question invites the person to be their authentic } \\
\text { self - and you will listen. This experience can be } \\
\text { transformational. }\end{array}$ \\
\hline
\end{tabular}


Lown argues the systemic factors and 'barriers that impede connection, caring and compassion must be addressed so that caregivers can offer, and patients and families receive, compassionate, patient and family centred care. Compassionate care must be a fundamental element of our healthcare system - available to all patients and families every day and in every interaction' (2014, p.12).

The elements of compassion (Cornwell \& Goodrich, 2009, p. 8)

- Compassion starts with good basic care and can be demonstrated in very practical ways, e.g. ensuring a patient's feeding needs are addressed, that pain is managed and the patient is helped to the bathroom.

- Compassion goes beyond this, to encompass empathy, respect, a recognition of the uniqueness of another individual and willingness to enter into a relationship in which not only the knowledge but the intuitions, strengths, and emotions of both patient and [caregiver] can be fully engaged.

- Compassion involves 'real dialogue'.

- The compassionate caregiver never stereotypes but appreciates difference, recognising the common humanity shared by both patient and caregiver.

- Compassion should not be seen as being sweet and nice. It includes honesty and may require courage.

- It is not a one-size-fits-all approach. Compassion can mean very different things in different situations and to different people. In recognising the individuality of each patient, compassionate carers will also recognise how best to tailor their behaviour to show compassion based on an individual's needs.

- Compassion means seeing the person in the patient at all times and all points of care.

Key Readings:

Adamson, L. \& Dewar, B. (2014). Compassionate care: Student nurses' learning through reflection and the use of story. Nurse Education in Practice, 15(3), 155-161.

van der Cingel, M. (2014). Compassion: The missing link in quality of care. Nurse Education Today, 34(9), 12531257.

Davidson, P., Daly, J. \& Hill, M. (2013). Looking to the future with courage, commitment, competence and compassion. Journal of Clinical Nursing, 22(19-20), 2665-2667.

\section{POST-INTERVENTION QUALITATIVE QUESTIONS}

We are interested to know how undergraduate nursing students understand the topic of compassion in nursing after studying the online compassion module. We are inviting you to respond to fifteen open-ended questions in Part A. Please type your responses into the text box under each question. Part B consists of seven demographic questions. These questions should take no longer than fifteen to twenty minutes to complete. Your ideas are very important to us. By submitting your responses, you are consenting to participate in the study. Responses are confidential, and participation is voluntary. Responses are anonymous and will only be viewed by the researchers. Findings will be used to improve the teaching of this topic to future undergraduate nursing students. This study has received ethical approval from XX Review Board. Thank you for participating.

\section{Part A}

1. What does compassion mean to you now?

2. What does practicing compassion mean to you now?

3. Can you give an example of you (or someone else) practising compassion toward a client, resident, or patient?

4. Why do you think it is a good example?

5. Can you give an example of you (or someone else) practising compassion toward a colleague in the workplace?

6. Why do you think it is a good example?

7. Can you describe a situation when you felt that compassion toward a patient or colleague was missing?

8. What do you think hinders nurses from delivering compassionate care (barriers)? 
9. What do you think would help nurses to be more compassionate in the care they give?

10. Why do you think it is important to be resilient as a nurse?

11. To take care of others, we have to take care of ourselves. What can you do to build your resilience?

12. What is one important idea that you have learnt in the compassion module?

13. Thinking about what you have learnt, how could this knowledge influence your practice as a nurse?

14. How could the compassion module be improved - what other content do you think should be included?

15. What six (6) words summarize this topic for you?

\section{Part B}

1. Are you: $17-21$ years; $22-29$ years; $30-39$ years; 40 - 49 years; $50-59$ years; other - please specify.

2. What is your gender: female; male

3. Are you enrolled as an international student: yes; no

4. What language do you speak most commonly at home?

5 . In what country were you born?

6. If you were not born in this country, in what year did you arrive?

7. What is your highest qualification? 


\section{NOTES}

